\title{
Nachsorge, Komplikationen und deren Therapie bei der operativen, strahlentherapeutischen und medikamentösen Therapie des Lungenkarzinoms
}

\author{
Axel Armbrecht ${ }^{\mathrm{a} *}$ Andreas Gröschel ${ }^{\mathrm{b} *}$ Christoph Pöttgen ${ }^{\mathrm{c} *}$ Martin Reck ${ }^{\mathrm{d} *}$ \\ Jens Ulrich Rüffer ${ }^{\mathrm{e} *}$ Ulf Seifart ${ }^{\mathrm{f} *}$ Cornelia M. Ulrich ${ }^{\mathrm{g*}}$ Hauke Winter ${ }^{\mathrm{h} *}$

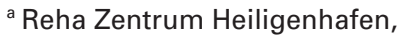 \\ ${ }^{\mathrm{b}}$ Klinik für Innere Medizin V/Pneumologie, Universitätsklinikum des Saarlandes, Homburg/Saar, \\ ${ }^{c}$ Klinik für Strahlentherapie, Universitätsklinikum Essen, \\ dOnkologischer Schwerpunkt, Krankenhaus Großhansdorf, \\ ${ }^{e}$ Deutsche Fatigue Gesellschaft e.V., Köln, \\ ${ }^{f}$ Rehabilitationsklinik für Onkologie und Orthopädie, Klinik Sonnenblick, Marburg, \\ ${ }^{g}$ Abteilung Präventive Onkologie, Nationales Zentrum für Tumorerkrankungen Heidelberg, \\ ${ }^{\text {h }}$ Chirurgische Klinik und Poliklinik, Klinikum der Universität München - Großhadern, Deutschland
}

Komplikationen nach chirurgischer Therapie:

Stumpfinsuffizienz und Pleuraempyem

\section{Postoperative bronchopleurale Fisteln}

Nach Resektion eines nichtkleinzelligen Lungenkarzinoms (NSCLC) entwickeln bis zu 15\% der Patienten bronchopleurale Fisteln. Die Häufigkeit der mit einer hohen Morbidität und Mortalität einhergehenden Komplikationen korreliert mit der Größe des Eingriffs (Pneumonektomie $>$ Manschettenresektion $>$ Lobektomie) und ist bei rechtsseitigen Eingriffen größer als bei linksseitigen. Als wichtiger Risikofaktor für die Entwicklung einer Stumpfinsuffizienz nach Pneumonektomie wurde neben einem rechtsseitigen Eingriff eine prolongierte postoperative Beatmung identifiziert [1].

Die Therapie postoperativer bronchopleuraler Fisteln erfolgt in Abhängigkeit von der Größe sowie der Entwicklung eines Pleuraempyems. Kleine Fisteln mit einem Durchmesser $\leq 5 \mathrm{~mm}$ ohne Empyem können gegebenenfalls bronchoskopisch mittels Fibrinkleber versorgt werden. Diese Vorgehensweise ist unabhängig von der Fistelgröße auch bei allen nicht operablen Patienten eine Therapieoption. Bei größeren Fisteln, Pleuraempyem oder Versagen der initialen interventionellen Therapie wird eine Operation mit Verschluss der bronchopleuralen Fistel, Deckung mit gestielten Muskellappen (M. Serratus, M. Latissimus dorsi, M. Pectoralis, Omentum majus), Dekortikation der Lunge und Einlage von Spüldrainagen empfohlen.

*Alle Autoren haben zu gleichen Teilen zu diesem Artikel beigetragen.
Post-Pneumonektomie-Pleuraempyem

Die Inzidenz eines Pleuraempyems nach Pneumonektomie liegt Literaturangaben zufolge zwischen 0,8 und $15 \%$. In einer USamerikanischen Studie waren vor allem folgende Faktoren mit der Entwicklung eines Empyems assoziiert [2]:

- Tumor $(p=0,0001)$

- präoperativ eingeschränkte Einsekundenkapazität $\left(\mathrm{FEV}_{1}\right)$ $(\mathrm{p}<0,01)$

- präoperativ erniedrigte Diffusionskapazität (DLCO) $(\mathrm{p}=$ 0,0001)

- rechtsseitige Pneumonektomie $(p=0,0109)$

- Komplettierungspneumonektomie $(p<0,01)$

- Bluttransfusionen $(\mathrm{p}<0,01)$

Die stadiengerechte Therapie des ebenfalls mit einer hohen Morbidität und Mortalität assoziierten Pleuraempyems reicht von der Thoraxdrainage bei einem Frühempyem über videoassistierte Thorakoskopie (VATS) bis hin zur Thorakoplastik (Abb. 1) [3]. Zur Sanierung der infizierten Thoraxhöhle kann die Einlage eines Vacuum Assisted Closure (VAC)-Schwammes oder eines gestielten Muskellappens notwendig sein.

Nach Strahlentherapie: Pneumonitis und

Zentralnervensystem-Komplikationen

\section{Strahlenpneumonitis}

Nach Strahlentherapie eines NSCLC kommt es bei zahlreichen Patienten zu einer akuten Zytokin-vermittelten Pneumonitis, zum Teil mit chronischer Lungenfibrose. Die Inzidenz schwerer,

\section{KARGER}

Fax +497614520714

Information@Karger.de

www.karger.com (c) 2011 S. Karger GmbH, Freiburs

0378-584X/11/3416-0011\$38.00/0 


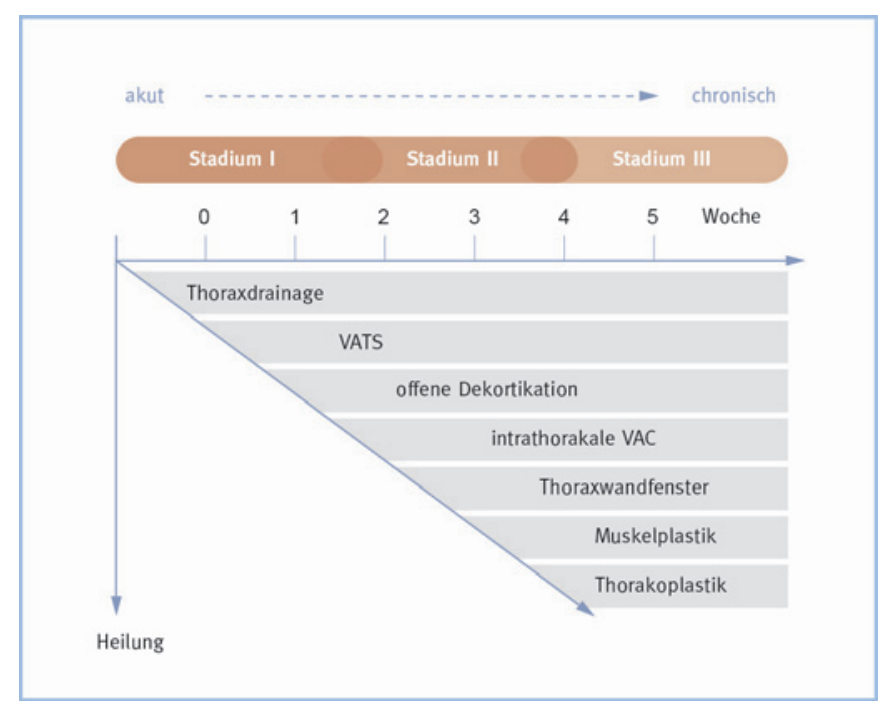

Abb. 1. Stadienadaptierte Therapie des Pleuraempyems nach Pneumonektomie (modifiziert nach [3]).

symptomatischer Pneumonitiden ( $\mathrm{Grad} \geq 3$ ), die zu relevanten Einschränkungen der Aktivitäten des täglichen Lebens (ADL) führen können, beträgt Literaturangaben zufolge 10-20\%. Basierend auf der Radiation Therapy Oncology Group (RTOG)-Studie 9311 und Daten der Universität Washington $(\mathrm{n}=324)$ wurde ein Nomogramm entwickelt, mit dem das individuelle Pneumonitis-Risiko auf der Basis der Lungendosis (mean lung dose, MLD) und der Tumorlokalisation abgeschätzt werden kann [4].

Um bei Patienten mit NSCLC die Pneumonitis-Rate auf $<20 \% \mathrm{zu}$ reduzieren, sollte bei der definitiven Bestrahlung das Lungenvolumen, das eine Dosis $\geq 20$ Gy erhält $\left(\mathrm{V}_{20}\right)$, auf $\leq 30$ $35 \%$ und die MLD auf $\leq 20-23$ Gy (bei konventioneller Fraktionierung) begrenzt werden. Sollte nach Pneumonektomie eine Strahlentherapie vorgesehen werden, sollten das $V_{20}$ auf $<4-10 \%$, das $\mathrm{V}_{5}$ auf $<60 \%$ und die MLD auf $<8$ Gy begrenzt werden [5].

Die therapeutischen Optionen bei Strahlenpneumonitis sind begrenzt. Im Vordergrund steht die Gabe von Steroiden (Prednison $\geq 50-100 \mathrm{mg} / \mathrm{d}, 1-2$ Wochen, danach ausschleichend), bei respiratorischer Insuffizienz ist die Gabe von Sauerstoff erforderlich [6]. Der therapeutische Stellenwert von Antibiotika ist vom Einzelfall abhängig.

\section{Zentralnervensystem-Komplikationen}

Patienten mit NSCLC im Stadium II-IIIB haben ein hohes Risiko für Hirnmetastasen, die durch eine adjuvante Chemotherapie nicht wesentlich verhindert werden können. Während bei multiplen Hirnmetastasen eine Ganzhirnbestrahlung indiziert ist, können singuläre Hirnmetastasen radiochirurgisch oder operativ behandelt werden. Ob eine adjuvante Ganzhirnbestrahlung unmittelbar angeschlossen werden sollte, ist insbesondere nach stereotaktischer Bestrahlung Gegenstand der Debatte.

In einer Studie der European Organisation for Research and Treatment of Cancer (EORTC), in der nach stereotaktischer Radiochirurgie sequenziell eine Ganzhirnbestrahlung durchgeführt wurde (vs. keine weitere Strahlentherapie), verringerte die adjuvante Ganzhirnbestrahlung zwar das Risiko einer intrakraniellen Progression, die zusätzliche Ganzhirnbestrahlung beeinträchtigte allerdings auch physische und kognitive Funktionen [7].

Bestätigt wurden diese Daten durch die Ergebnisse einer randomisierten Studie bei Patienten mit 1-3 neu diagnostizierten Hirnmetastasen $(\mathrm{n}=58)$ und bereits bei Studienbeginn eingeschränkten neurokognitiven Funktionen. Bei Patienten, bei denen nach stereotaktischer Radiochirurgie anschließend eine Ganzhirnbestrahlung durchgeführt wurde, wurde nach 4 Monaten eine deutlich stärkere Abnahme kognitiver Leistungen beobachtet als bei Patienten mit alleiniger Radiochirurgie (52\% vs. 24\%) [8].

$\mathrm{Ob}$ sich strahlenbedingte neurokognitive Defizite durch selektive Schonung neuronaler Stammzellbereiche vermeiden lassen, wird in ersten Machbarkeitsstudien untersucht [9]. Eine Therapie radiogener Komplikationen muss individuell optimiert werden; neben Steroiden (Dexamethason) werden in Einzelfällen auch weitere anti-ödematös wirksame Substanzen eingesetzt. Symptomatische Nekrosen nach Radiochirurgie können einen operativen Eingriff notwendig machen. Bei Patienten mit demenziellem Syndrom können in Einzelfällen Anticholinergika indiziert sein.

\section{Chemotherapie: Nebenwirkungen an Niere, Knochenmark, Lunge und peripherem Nervensystem}

Nebenwirkungen einer Chemotherapie manifestieren sich in den meisten Fällen während der Behandlung, können wie periphere Neuropathien (PNP) durch Vinca-Alkaloide, Platinderivate oder Taxane aber auch erst einige Wochen oder Monate nach Behandlungsende auftreten bzw. sich verstärken.

\section{Renale Nebenwirkungen}

Ein häufig unterschätztes Problem bei der Chemotherapie der überwiegend älteren Tumorpatienten ist die Nephrotoxizität der verabreichten Substanzen, die durch weitere Schädigungsmechanismen verstärkt werden kann. Neben einem intravasalen Volumenmangel (Wasserverlust oder Verlust in andere Kompartimente z.B. bei Aszites, Pleuraerguss) stellen vor allem die Toxizität der Begleitmedikation (z.B. Aminoglykoside, Analgesie mit nichtsteroidalen Antirheumatika (NSAR), Kontrastmittelgabe im Rahmen von Staging-Untersuchungen) und primäre Nierenerkrankungen ein erhöhtes Nephrotoxizitätsrisiko dar.

In einer französischen Beobachtungsstudie, in der die Daten von über 4600 Patienten mit soliden Tumoren ausgewertet wurden, hatten zwar nur 7,2\% der Patienten erhöhte Serumkreatininwerte, der Anteil der Patienten mit Einschränkungen der Kreatinin-Clearance (Cockcroft-Gault $\mathrm{CrCl}<90 \mathrm{ml} / \mathrm{min}$ ) lag aber über 50\%. In der Subgruppe der Patienten mit Lungenkarzinom $(\mathrm{n}=445)$ betrug der Anteil der Patienten mit einer Kreatinin-Clearance $<90 \mathrm{ml} / \mathrm{min} 62 \%$ (Tab. 1) [10].

Hinweise zur individuellen Dosierung von Zytostatika und anderen Arzneimitteln bei Patienten mit eingeschränkter Nierenfunktion sowie Daten zu unerwünschten Ereignissen (UAW) an Nieren und Harnwegen und den bei niereninsuffizienten Patien- 
Tab. 1. Nierenfunktion von Patienten mit Lungenkarzinom $(n=445)$

\begin{tabular}{lcc}
\hline Cockcroft-Gault $\mathrm{CrCl}, \mathrm{ml} / \mathrm{min}$ & $\mathrm{n}$ & $\%$ \\
\hline$\geq 90$ & 138 & 31,0 \\
$89-60$ & 172 & 38,6 \\
$59-30$ & 95 & 21,4 \\
$29-15$ & 9 & 2,0 \\
$<15$ & 0 & 0 \\
Nicht bestimmt & 31 & 7,0 \\
\hline
\end{tabular}

Modifiziert nach [10].

ten beobachteten UAW werden in einer vom Universitätsklinikum Heidelberg erstellten und kontinuierlich aktualisierten Datenbank zur Verfügung gestellt [11].

\section{Anämie}

Chemotherapie-induzierte Anämien werden auch bei Patienten mit Lungenkarzinom relativ häufig beobachtet. Nach Ausschluss bzw. Korrektur anderer Ursachen einer Anämie, wie Blutungen, Eisenmangel, Hämolyse, Nierenfunktionsstörungen oder hämatologischen Systemerkrankungen, können symptomatische Patienten mit Hämoglobin (Hb)-Werten zwischen 9 und $11 \mathrm{~g} / \mathrm{dl}$ bis zu einem Zielwert von $12 \mathrm{~g} / \mathrm{dl}$ Erythropoese stimulierende Agenzien (ESA) wie Erythropoetin- $\alpha$ oder $-\beta$ bzw. Darbepoetin erhalten. Die Vor- und Nachteile (Thrombosen, verkürzte Lebensdauer) sollten gegen eine Transfusion abgewogen werden. Es wird empfohlen, die ESA-Therapie nach Beendigung der Chemo- bzw. Strahlentherapie nicht fortzusetzen [12].

\section{Pulmonale Toxizitäten}

Der Nachweis Chemotherapie-induzierter pulmonaler Toxizitäten kann sich bei Lungenkarzinom-Patienten schwierig gestalten, da es sich hierbei meist um unspezifische, nach unterschiedlichem Muster ablaufende Parenchymveränderungen handelt. Erschwerend kommt hinzu, dass viele Patienten Vorerkrankungen wie chronisch obstruktive Lungenerkrankungen (COPD) oder Emphyseme aufweisen und auch der Tumor selbst die Lungenfunktion verschlechtern kann. Neben einer vorbestehenden interstitiellen Lungenerkrankung, höherem Alter, Nikotinkonsum, renaler Dysfunktion und Sauerstofftherapie wurde vor allem die kumulative Dosis des applizierten Chemotherapeutikums als Risikofaktor beschrieben. Eine begleitende Strahlentherapie kann die Beschwerden verstärken [13].

Angaben zur Art und Häufigkeit Arzneimittel-induzierter Lungentoxizitäten finden sich in einer von der französischen GEPPI (Groupe d'Etudes de la Pathologie Pulmonaire Iatrogène) erstellten Datenbank [14].

\section{Periphere Neuropathie}

Die Inzidenz der Chemotherapie-induzierten peripheren Neuropathie (CIPN) wird bei einer neurotoxischen Monotherapie auf 3-7\% geschätzt und kann bei Kombinationschemotherapien bis zu $38 \%$ betragen. Da die Nervenschädigungen nur langsam bzw. teilweise nicht vollständig reversibel sind und bisher keine Standardtherapie verfügbar ist, wurden zur Prophylaxe und Therapie der CIPN auch verschiedene komplementärmedizinische Therapieansätze untersucht. Eine neuroprotektive Wirkung von Calcium-/Magnesium-Infusionen, Glutamin/Glutathion, L-Carnitin, Akupunktur und Vitamin E konnte bisher allerdings nur in kleineren Studien gezeigt werden, wobei die Ergebnisse teilweise widersprüchlich waren.

\section{Targeted Therapies: Nebenwirkungen und Perspektiven}

Inhibitoren des epidermalen Wachstumsfaktorrezeptors (EGFR) und des vaskulären endothelialen Wachstumsfaktors (VEGF) haben die Prognose beim fortgeschrittenen NSCLC deutlich verbessert. Verglichen mit konventionellen Chemotherapeutika fallen bei den bisher zugelassenen Substanzen allerdings einige neue Nebenwirkungen auf, die den Einsatz begrenzen, aber auch zur Selektion der von diesen Therapieansätzen voraussichtlich besonders profitierenden Patienten genutzt werden können.

\section{EGFR-Inhibition}

Charakteristische Nebenwirkungen der beim fortgeschrittenen NSCLC zugelassenen EGFR-Tyrosinkinase-Inhibitoren (EGFRTKI) Erlotinib (Tarceva ${ }^{\circledR}$, Roche Pharma AG) und Gefitinib (Iressa ${ }^{\circledR}$, Astra Zeneca $\mathrm{GmbH}$ ) sind Hautreaktionen, vor allem ein in den ersten Therapiewochen auftretender, überwiegend leichter oder moderater Rash, sowie meist gut kontrollierbare Diarrhöen. Pulmonale Toxizitäten wurden bei asiatischen Patienten, die mit Gefitinib behandelt wurden, mit einer Inzidenz von 2,6-5,3\% beschrieben (IPASS, WJOG) $[15,16]$. Bei kaukasischen Patienten wurden Pneumonitiden mit einer Inzidenz $<1 \%$ (Erlotinib: BR.21, SATURN, TRUST) bzw. 1\% (Gefitinib: INTEREST) dagegen selten gesehen [17-20].

Die Bedeutung eines EGFR-Inhibitor-induzierten papulopustulösen Exanthems für das Therapieansprechen zeigte unter anderem eine retrospektive Analyse der BR.21-Studie, in der chemotherapeutisch vorbehandelte Patienten, die unter Erlotinib Rash Grad > 1 entwickelten, signifikant länger lebten als Patienten ohne diese Hautreaktion (11,1 vs. 3,3 Monate; Hazard Ratio (HR) 0,29; p < 0,001) [21]. Ein besseres klinisches Outcome bei Patienten mit Rash Grad $\geq 2$ zeigten auch die Ergebnisse der Phase-IV-Studie TRUST. In der 1-armigen Studie war die progressionsfreie Überlebenszeit (PFS) bei Patienten mit Rash Grad $\geq 2(\mathrm{n}=2649)$ mit median 5,5 Monaten etwa doppelt so lang wie bei Patienten mit Rash Grad 0/1 ( $\mathrm{n}=3390$; medianes PFS 2,6 Monate; HR 0,59; $\mathrm{p}<0,0001)$ [19].

Vor diesem Hintergrund bemerkenswert war die Beobachtung, dass im klinischen Alltag Raucher bei Einnahme von Erlotinib in der beim Lungenkarzinom zugelassenen Dosierung von 150 mg/d seltener Rash entwickeln als Nichtraucher oder Exraucher. Auf mögliche Wirksamkeitsunterschiede in Abhängigkeit vom Raucherstatus deuteten auch die Ergebnisse einer Phase-I/ II-Studie hin, in der chemotherapeutisch vorbehandelte Raucher ( $\geq 10$ Zigaretten/Tag, $\geq 1 \mathrm{Jahr}$ ) zunächst Erlotinib in eskalierenden Dosierungen von täglich 200 bis $350 \mathrm{mg}$ erhielten. Anschließend wurden die Pharmakokinetik und Wirksamkeit von Erlotinib in der maximal tolerablen Dosis von $300 \mathrm{mg} / \mathrm{d}$ und $150 \mathrm{mg} / \mathrm{d}$ prospektiv, randomisiert verglichen [22]. 


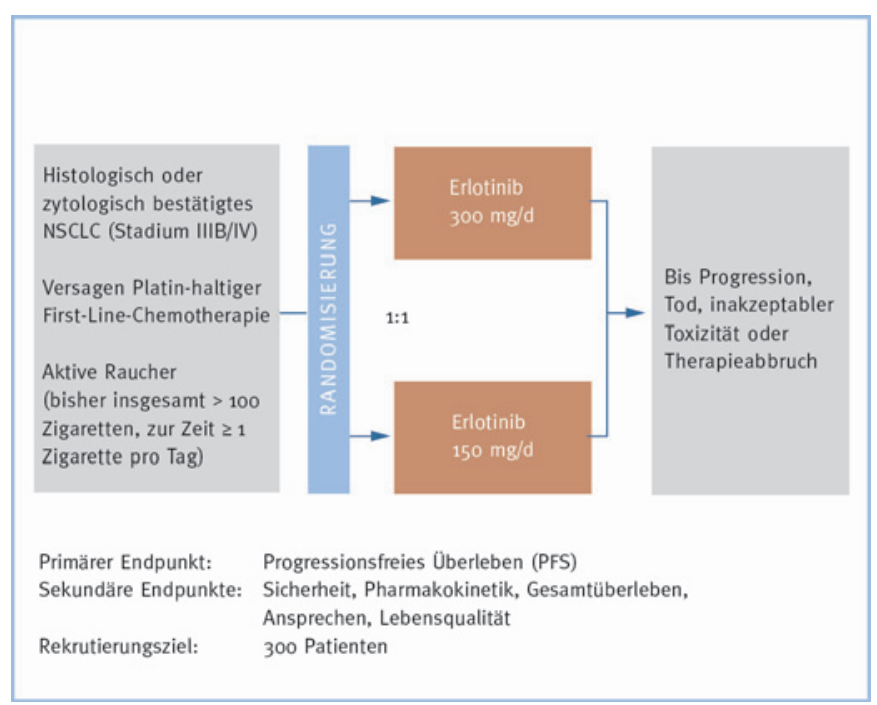

Abb. 2. Design der Phase-III-Studie CURRENTS.

Die Erlotinib-Plasmaspiegel und die Inzidenz an Hauttoxizitäten lagen bei den mit 300 mg/d behandelten Rauchern im selben Bereich wie dies in früheren Studien für Nichtraucher gezeigt worden war, die Erlotinib in der beim NSCLC zugelassenen Dosierung von $150 \mathrm{mg} / \mathrm{d}$ erhalten hatten [23]. Da in der Phase-I/ II-Studie das mediane Gesamtüberleben der Raucher, die mit Erlotinib in einer Dosierung von täglich 250 oder $300 \mathrm{mg}$ behandelt wurden, deutlich besser war als bei Einnahme in der beim NSCLC üblichen Dosierung von $150 \mathrm{mg} / \mathrm{d}$ (9,6 vs. 5,5 Monate), wurde die doppelblinde, randomisierte Phase-III-Studie CURRENTS initiiert, in der bei Rauchern mit fortgeschrittenem NSCLC und Versagen einer platinhaltigen Erstlinien-Chemotherapie die Wirksamkeit von Erlotinib in zwei Dosierungen (300 vs. $150 \mathrm{mg} / \mathrm{d}$ ) untersucht wird (Abb. 2).

\section{VEGF-Inhibition}

Charakteristische Nebenwirkungen einer VEGF-Inhibition sind Hypertonie, Proteinurie, Wundheilungsstörungen und Blutungsereignisse. Klinische oder radiologische Faktoren, die bei der Erstlinien-Therapie mit Bevacizumab (Avastin ${ }^{\circledR}$, Roche Pharma AG) ein erhöhtes Risiko für pulmonale Blutungen anzeigen, konnten bisher nicht identifiziert werden. Nach den vorliegenden Daten sind weder Alter, Geschlecht oder Allgemeinzustand noch eine vorherige Radiotherapie prädiktiv für ein erhöhtes pulmonales Blutungsrisiko. Frühere Hinweise auf ein erhöhtes Risiko bei initial bestehender Tumorkaverne wurden durch die Daten der Phase-IV-Studie ARIES, in der von 1881 Patienten 15 (0,8\%) eine schwere pulmonale Blutung entwickelten, nicht bestätigt [24].

Die Bedeutung einer Bevacizumab-induzierten Hypertonie für die Wirksamkeit des VEGF-Antikörpers ist noch unklar. Während in der Phase-III-Studie ECOG4599 im BevacizumabArm ein Blutdrucksanstieg unter der Therapie mit einem deutlich besseren Gesamtüberleben assoziiert war (15,9 vs. 11,5 Monate) [25], zeigte die systematische Analyse von 6 klinischen Stu-

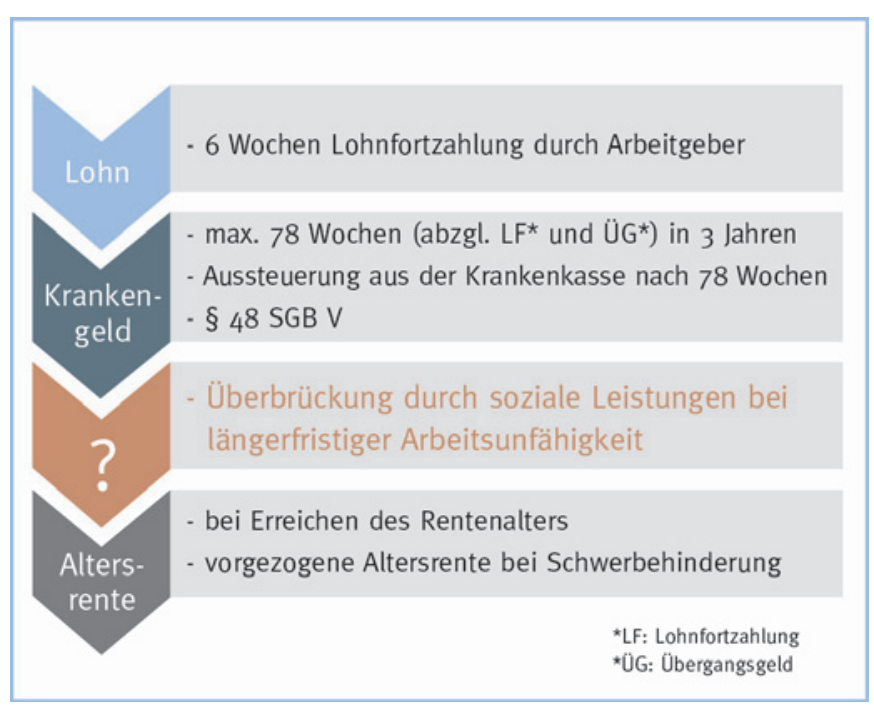

Abb. 3. Finanzielle Absicherung bei Arbeitsunfähigkeit.

dien, in denen die Daten von etwa 5900 Patienten mit unterschiedlichen Tumorentitäten ausgewertet wurden, keine Korrelation zwischen der Manifestation einer Hypertonie und dem PFS oder Gesamtüberleben [26].

\section{Sozialmedizinische Aspekte}

Die soziale Reintegration von Tumorpatienten scheint nicht nur für die Lebensqualität, sondern auch für das Gesamtüberleben bedeutsam zu sein. In der Nurses' Health Study wurde bei 2835 Brustkrebspatientinnen gezeigt, dass sozial nicht oder schlecht integrierte Frauen (evaluiert mittels Berkman-Syme Social Network Index) eine 2-fach erhöhte Brustkrebsmortalität aufwiesen [27]. Eine in Schweden bei Patienten mit akuter myeloischer Leukämie (AML) und multiplem Myelom (MM) durchgeführte Studie zeigte für Gruppen mit unterschiedlichem sozioökonomischen Status signifikante Unterschiede im Gesamtüberleben. Bei beiden hämatologischen Erkrankungen hatten höhere Angestellte eine statistisch signifikant bessere Prognose als Arbeiter (AML: $\mathrm{p}=$ 0,005; MM: p < 0,005) [28]. Da der soziale Status auch von der Arbeitsfähigkeit abhängt und überlebende Tumorpatienten einer großen Metaanalyse zufolge häufiger arbeitslos sind als gleichaltrige Gesunde (33,8\% vs. $25,2 \%$ ) [29], sollte bereits bei der Therapieplanung an mögliche Beeinträchtigungen der Arbeitsfähigkeit gedacht und frühzeitig entsprechende Schritte eingeleitet werden.

Bei Arbeitsunfähigkeit sind versicherungspflichtige Arbeitnehmer für maximal 78 Wochen durch Krankengeldzahlungen finanziell recht gut abgesichert (Abb. 3); Probleme sind aber immer dann zu erwarten, wenn die Arbeitsfähigkeit so weit eingeschränkt ist, dass der Patient nicht mehr in seinem ursprünglichen Beruf arbeiten kann. Hier sollte geprüft werden, ob die eingeschränkte Arbeitsfähigkeit durch Veränderungen am Arbeitsplatz kompensiert werden kann oder eine stufenweise Wiedereingliederung, beispielsweise nach dem Hamburger Modell, möglich ist. 
Tab. 2. Stufen der Verhaltensmodifikation nach dem Transtheoretischen Modell

\author{
Absichtslosigkeit («precontemplation») \\ Absichtsbildung («contemplation») \\ Vorbereitung («preparation») \\ Handlung («action») \\ Aufrechterhaltung («maintenance») \\ Stabilisierung («termination»)
}

keine Intention, das problematische Verhalten in nächster Zeit zu ändern

Erwägung, das problematische Verhalten in den nächsten

6 Monaten zu verändern

Einleitung erster Schritte zur Verhaltensänderung

Umsetzung der geplanten Verhaltensänderung in $<6$ Monaten

Beibehaltung des Zielverhaltens für $>6$ Monate

wie Aufrechterhaltung, jedoch ohne Rückfallgefahr

Modifiziert nach [31].

Falls eine Weiterbeschäftigung im ursprünglichen Beruf aus gesundheitlichen Gründen nicht mehr möglich ist, sollte auf Basis der körperlichen Leistungsfähigkeit und unter Berücksichtigung der bei Lungenkarzinom-Patienten häufig eingeschränkten Lungenfunktion sowie eventuell vorliegender Gefährdungs- und Belastungsfaktoren die Eignung für alternative Tätigkeiten geprüft werden. Patienten mit einer anerkannten Schwerbehinderung können hierfür auf die Hilfe des Integrationsfachdienstes zurückgreifen. Für alle Patienten stehen Hilfen der Arbeitsagentur und der Deutschen Rentenversicherung zur Verfügung. Detaillierte Informationen hierzu können Sozialdienste, Beratungsstellen, Selbsthilfegruppen, Rehabilitationsfachberater und nicht zuletzt Rehabilitationskliniken vermitteln.

Versicherungspflichtigen, die infolge der krankheitsbedingten Einschränkungen täglich weniger als 3 Stunden in ihrer zuletzt durchgeführten versicherungspflichtigen Tätigkeit und auf dem «Allgemeinen Arbeitsmarkt» arbeiten können, steht eine Erwerbsunfähigkeitsrente zu, wenn sie zuvor mindestens 5 Jahre lückenlos Beiträge in die Deutsche Rentenversicherung gezahlt haben.

\section{Psychoonkologische Interventionen}

Bei der Mehrzahl der Tumorpatienten liegt eine klinisch relevante psychosoziale Belastung vor, die häufig auch nach Abschluss der Therapie andauert. Jeder 2. bis 4. Patient leidet unter einer Angststörung oder Depression. In den meisten Fällen handelt es sich um behandlungsbedürftige Veränderungen, die effektiv durch psychoonkologische Interventionen therapiert werden können. Die Zielbereiche der auch in der interdisziplinären S3-Leitlinie zur Prävention, Diagnostik, Therapie und Nachsorge des Lungenkarzinoms empfohlenen psychoonkologischen Interventionen umfassen neben Depression, Angst und Krankheitsverarbeitung unter anderem den Umgang mit belastenden Therapienebenwirkungen (z.B. Übelkeit und Erbrechen, neurokognitive Beeinträchtigungen), Schmerzen und Fatigue sowie Veränderungen in Körperbild und Sexualität [30]. Grundlage der 4-stufigen Behandlung, bei der der Psychoonkologe in vielen Bereichen mit den primär behandelnden Ärzten zusammenarbeitet, ist eine offene Kommunikation mit dem Patienten.

\section{Lebensstiländerungen}

Ein weiterer Schwerpunkt psychoonkologischer Interventionen ist die Unterstützung des Patienten bei der Änderung gesundheitsschädlicher Verhaltensweisen, beispielsweise Rauchen, Alkoholmissbrauch, Überernährung und Bewegungsmangel. Nach dem Transtheoretischen Modell wird die Verhaltensänderung als stufenförmig verlaufender Prozess verstanden, wobei jede der empirisch identifizierten Stufen durch etwa 13 kognitiv-affektive und verhaltensorientierte Konstrukte definiert ist (Tab. 2) [31].

\section{Sport und Krankheitsbewältigung}

Die bisher vorliegenden Studiendaten [32] deuten darauf hin, dass Tumorpatienten sowohl während der Therapie als auch nach Therapieabschluss von systematischer sportlicher Betätigung profitieren. Zwar streuen die Ergebnisse in Abhängigkeit von der Tumorart und Dauer der sportlichen Belastung, doch findet die Aufnahme von Sport als therapeutischem Mittel zunehmend Anerkennung. Neben der Unterstützung des Heilungsprozesses durch Verbesserung der Durchblutung, Ventilation und Einfluss auf verschiedene Biomarker (z.B. Entzündung, Glucose-InsulinStoffwechsel) können regelmäßiges Ausdauertraining und Krafttraining die Fatigue reduzieren, das Selbstbewusstsein der Patienten stärken und auf diese Weise zur Krankheitsbewältigung beitragen. Das Training in einer Gruppe verbessert zudem die Sozialkontakte und führt den Patienten aus der Passivität des Therapieerlebens.

Um das Potenzial sportlicher Aktivitäten auf den Therapieerfolg besser einschätzen zu können, sind weitere Studien nötig, die konsequenter als bisher die Erkenntnisse der Sportwissenschaften in die therapeutischen Strategien einbeziehen. So sind neben den meist wöchentlichen Gruppenkontakten Programme zu erstellen, die tägliche Trainingsmodule sowohl im Ausdauerals auch im Krafttrainingsbereich beinhalten, orientiert am Prinzip der Superkompensation. Eine hohe Trainingsfrequenz von wenigen Minuten Dauer lässt auch bei Tumorpatienten größere Erfolge erwarten.

\section{Nahrungsergänzungsmittel}

Die systematische Auswertung von 32 Studien bei US-amerikanischen erwachsenen Tumorpatienten zeigte, dass 64-81\% Mineralstoff- oder Vitaminpräparate einnahmen, wobei 14-32\% damit erst nach Diagnosestellung begonnen hatten. Besonders hoch war 
die Rate bei Frauen und Patienten mit höherem Bildungsniveau [33]. Als Argumente für die Einnahme von Nahrungsergänzungsmitteln wurden in verschiedenen Studien unter anderem genannt:

- Empfehlung des medizinischen Personals

- Verbesserung der Befindlichkeit

- Stressbewältigung

- Stärkung des Immunsystems

- Erhöhung der Heilungschancen

- sichere Abdeckung des Ernährungsbedarfs

Die zur Wirksamkeit von Nahrungsergänzungsmitteln bei Tumorpatienten erhobenen Daten reichen bisher aber nicht aus, um fundierte Empfehlungen aussprechen zu können. Bedenken gibt es unter anderem bezüglich möglicher Interaktionen zwischen Standardtherapien und Nahrungsergänzungsmitteln (z.B. Johanniskraut) sowie, dass manche Vitaminpräparate (z.B. Folsäure) schädlich sein könnten. Problematisch ist darüber hinaus, dass viele Ärzte über die Einnahme von Nahrungsergänzungsmitteln nicht informiert sind.

\section{Disclosure Statement}

Die Autoren waren Referenten beim «Dresdner Interdisziplinäres Gespräch», das von der Roche Pharma AG unterstützt wurde.

\section{Literatur}

$>_{1}$ Sirbu H, Busch T, Aleksic I, et al.: Bronchopleural fistula in the surgery of non-small cell lung cancer: incidence, risk factors, and management. Ann Thorac Cardiovac Surg 2001;7:330-336.

2 Deschamps C, Bernard A, Nichols FC 3rd, et al.: Empyema and bronchopleural fistula after pneumonectomy: factors affecting incidence. Ann Thorac Surg 2001;72:243-247.

3 Eichhorn ME, Winter H, Preissler G, et al.: Stadienadaptierte moderne Therapie des Pleuraempyems. Zentralbl Chir 2011;136:34-41.

$\checkmark 4$ Bradley JD, Hope A, El Naqa I, et al.: A nomogram to predict radiation pneumonitis, derived from a combined analysis of RTOG 9311 and institutional data. Int J Radiat Oncol Biol Phys 2007;69: 985-992.

5 Marks LB, Bentzen SM, Deasy JO, et al.: Radiation dose-volume effects in the lung. Int J Radiat Oncol Biol Phys 2010;76(suppl):S70-S76.

$\checkmark 6$ Berkey FJ: Managing the adverse effects of radiation therapy. Am Fam Physician 2010;82:381-388, 394.

7 Mueller RP, Soffietti R, Abacioglu MU, et al.: Adjuvant whole-brain radiotherapy versus observation after radiosurgery or surgical resection of 1-3 cerebral metastases: Results of the EORTC 22952-26001 study. J Clin Oncol 2009;27(suppl 15s):abstr 2008

8 Chang EL, Wefel JS, Hess KR, et al.: Neurocognition in patients with brain metastases treated with radiosurgery or radiosurgery plus whole-brain irradiation: a randomised controlled trial. Lancet Oncol 2009;10:1037-1044.

-9 Barani IJ, Cuttino LW, Benedict SH, et al.: Neural stem cell-preserving external-beam radiotherapy of central nervous system malignancies. Int J Radiat Oncol Biol Phys 2007;68:978-985.

10 Launay-Vacher V, Oudard S, Janus N, et al.: Prevalence of renal insufficiency in cancer patients and implications for anticancer drug management: the renal insufficiency and anticancer (IRMA) study. Cancer 2007;110:1376-1384.

11 DOSING - Hilfsmittel zur Arzneimittel-Anwendung und Sicherheit, $w w w$.dosing.de.

-12 Rizzo JD, Brouwers M, Hurley P, et al.: American Society of Clinical Oncology/American Society of Hematology clinical practice guideline update on the use of epoetin and darbepoetin in adult patients with cancer. J Clin Oncol 2010;28:4996-5010.
13 De Sanctis A, Taillade L, Vignot S, et al.: Pulmonary toxicity related to systemic treatment of nonsmall cell lung cancer. Cancer 2011, in press, doi: $101002 /$ cncr.25894.

14 The drug-induced lung diseases, www.pneumotox. com.

15 Mok TS, Wu YL, Thongprasert S, et al.: Gefitinib or carboplatin-paclitaxel in pulmonary adenocarcinoma. N Engl J Med 2009;361:947-957.

16 Maemondo M, Inoue A, Kobayashi K, et al.: Gefitinib or chemotherapy for non-small-cell lung cancer with mutated EGFR. N Engl J Med 2010;362: 2380-2388.

17 Shepherd A, Pereira JR, Ciuleanu T, et al.: Erlotinib in previously treated non-small-cell lung cancer. N Engl J Med 2005;353:123-132.

18 Cappuzzo F, Ciuleanu T, Stelmakh L, et al.: Erlotinib as maintenance treatment in advanced nonsmall-cell lung cancer: a multicentre, randomised, placebo-controlled phase 3 study. Lancet Oncol 2010;11:521-529.

19 Reck M, van Zandwijk N, Gridelli C, et al.: Erlotinib in advanced non-small cell lung cancer. Efficacy and safety findings of the global phase IV Tarceva Lung Cancer Survival Treatment study. J Thorac Oncol 2010;5:1616-1622.

20 Kim ES, Hirsh V, Mok T, et al.: Gefitinib versus docetaxel in previously treated non-small-cell lung cancer (INTEREST): a randomised phase III trial. Lancet 2008;372:1809-1818.

21 Wacker B, Nagrani T, Weinberg J, et al.: Correlation between development of rash and efficacy in patients treated with the epidermal growth factor receptor tyrosine kinase inhibitor erlotinib in two large phase III studies. Clin Cancer Res 2007;13: 3913-3921.

22 Hughes AN, O'Brien MER, Petty WJ, et al.: Overcoming CYP1A1/1A2 mediated induction of metabolism by escalating erlotinib dose in current smokers. J Clin Oncol 2009;27:1220-1226.

23 Clark GM, Zborowski DM, Santabarbara P, et al.: Smoking history and epidermal growth factor receptor expression as predictors of survival benefit from erlotinib for patients with non-small-cell lung cancer in the National Institute of Canada Clinical Trials Group study BR.21. Clin Lung Cancer 2006; 7:389-394
24 Kumar P, Fischbach NA, Brahmer JR, et al.: Baseline radiographic characteristics and severe pulmonary hemorrhage in bevacizumab-treated nonsmall cell lung cancer patients: results from ARIES, an observational cohort study. J Thorac Oncol 2010;5(suppl 1):S62.

25 Dahlberg SE, Sandler AB, Brahmer JR, et al.: Clinical course of advanced non-small-cell lung cancer patients experiencing hypertension during treatment with bevacizumab in combination with carboplatin and paclitaxel in ECOG 4599. J Clin Oncol 2010;28:949-954.

26 Hurwitz H, Douglas PS, Middleton JP, et al.: Analysis of early hypertension (HTN) and clinical outcome with bevacizumab. J Clin Oncol 2010;28(suppl 15s):abstr 3039.

27 Kroenke CH, Kubzansky LD, Schernhammer ES et al.: Social networks, social support, and survival after breast cancer diagnosis. J Clin Oncol 2006;24:1105-1111.

28 Kristinsson SY, Derol AR, Edgren G, et al.: Socioeconomic differences in patient survival are increasing for acute myeloid leukemia and multiple myeloma in Sweden. J Clin Oncol 2009;27:20732080.

29 De Boer AG, Taskila T, Ojajärvi A, et al.: Cancer survivors and unemployment: a meta-analysis and meta-regression. JAMA 2009;301:753-762.

30 Goeckenjan G, Sitter H, Thomas M, et al.: Prävention, Diagnostik, Therapie und Nachsorge des Lungenkarzinoms. Pneumologie 2010;64(suppl 2):S23S155.

31 Keller S, Kaluza G, Basler HD: Motivierung zur Verhaltensänderung - Prozessorientierte Patientenedukation nach dem Transtheoretischen Modell der Verhaltensänderung. Psychomed 2001;13:101111.

32 Schmitz KH, Courneya KS, Matthews C, et al.: American College of Sports Medicine round table on exercise guidelines for cancer survivors. Med Sci Sports Exerc 2010;42:1409-1426.

33 Velicer CM, Ulrich CM: Vitamin and mineral supplement use among US adults after cancer diagnosis: a systematic review. J Clin Oncol 2008;26:665673. 